\title{
A signature of tumor DNA repair genes associated with the prognosis of surgically-resected lung adenocarcinoma
}

\author{
Xiongtao Yang ${ }^{1}$, Guohui Wang ${ }^{2}$, Runchuan Gu $^{3}$, Xiaohong Xu ${ }^{1}$, Guangying Zhu ${ }^{\text {Corresp. } 4}$ \\ ${ }^{1}$ Department of Radiation Oncology, Peking University China-Japan Friendship School of Clinical Medicine, Beijing, China \\ 2 Department of Radiotherapy, Second hospital of Hebei Medical University, Shijiazhuang, Hebei, China \\ 3 Department of Radiation Oncology, China-Japan Friendship Institute of Clinical Medicine, Beijing, China \\ 4 Department of Radiation Oncology, China-Japan Friendship Hospital, Beijing, China \\ Corresponding Author: Guangying Zhu \\ Email address: zryyfa@163.com
}

Background. Lung cancer has the highest morbidity and mortality of cancers worldwide. Lung adenocarcinoma (LUAD) is the most common pathological subtype of lung cancer and surgery is its most common treatment. The dysregulated expression of DNA repair genes is found in a variety of cancers and has been shown to affect the origin and progression of these diseases. However, the function of DNA repair genes in surgically-treated LUAD is unclear.

Methods. We sought to determine the association between the signature of DNA repair genes for patients with surgical LUAD and their overall prognosis. We obtained gene expression data and corresponding clinical information of LUAD from The Cancer Genome Atlas (TCGA) database. The differently expressed DNA repair genes of surgically-treated LUAD and normal tissues were identified using the Wilcoxon rank-sum test. We used uni- and multivariate Cox regression analyses to shrink the aberrantly expressed genes, which were then used to construct the prognostic signature and the risk score formula associated with the independent prognosis of surgically-treated LUAD. We used Kaplan-Meier and Cox hazard ratio analyses to confirm the diagnostic and prognostic roles. Two validation sets (GSE31210 and GSE37745) were downloaded from the Gene Expression Omnibus (GEO) and were used to externally verify the prognostic value of the signature. OSluca online database verifies the hazard ratio for the DNA repair genes by which the signature was constructed. We investigated the correlation between the signature of the DNA repair genes and the clinical parameters. The potential molecular mechanisms and pathways of the prognostic signature were explored using Gene Set Enrichment Analysis (GSEA).

Results. We determined the prognostic signature based on 6 DNA repair genes (PLK1, FOXM1, PTTG1, $\mathrm{CCNO}, \mathrm{HIST} 3 \mathrm{H} 2 \mathrm{~A}$, and BLM) and calculated the risk score based on this formula. Patients with surgicallytreated LUAD were divided into high-risk and low-risk groups according to the median risk score. The high-risk group showed poorer overall survival than the low-risk group; the signature was used as an independent prognostic indicator and had a greater prognostic value in surgically-treated LUAD. The prognostic value was replicated in GSE31210 and GSE37745. OSluca online database analysis shows that 6 DNA repair genes were associated with poor prognosis in most lung cancer datasets. The prognostic signature risk score correlated with the pathological stage and smoking status in surgically-treated LUAD. The GSEA of the risk signature in high-risk patients showed pathways associated with the cell cycle, oocyte meiosis, mismatch repair, homologous recombination, and nucleotide excision repair.

Conclusions. A 6-DNA repair gene signature was determined using TCGA data mining and GEO data

PeerJ reviewing PDF | (2020:05:48835:2:1:NEW 29 Oct 2020) 
verification. The gene signature may serve as a novel prognostic biomarker and therapeutic target for surgically-treated LUAD. 
1 A signature of tumor DNA repair genes associated

2 with the prognosis of surgically-resected lung

3 adenocarcinoma

4 Xiongtao Yang ${ }^{1}$, Guohui Wang ${ }^{2}$, Runchuan $\mathrm{Gu}^{3}$, Xiaohong $\mathrm{Xu}^{1}$, Guangying $\mathrm{Zhu}^{4}$

5 'Department of Radiation Oncology, Peking University China-Japan Friendship School of

6 Clinical Medicine, Beijing, China

7 2Department of Radiotherapy, Second Hospital of Hebei Medical University, Shijiazhuang,

8 Hebei, China

$9{ }^{3}$ Department of Radiation Oncology, China-Japan Friendship Institute of Clinical Medicine,

10 Beijing, China

$11{ }^{4}$ Department of Radiation Oncology, China-Japan Friendship Hospital, Beijing, China

12 Corresponding Author: Guangying Zhu

13 Yinghua East Street, Chaoyang District, Beijing, 100000, China

14 Email address: zryyfa@163.com

15

16

17

18

19

20

21

22

23

24

25

26

27

28

29

30

31

32

33

34

35

36

37

38

39

\section{Abstract}

Background. Lung cancer has the highest morbidity and mortality of cancers worldwide. Lung adenocarcinoma (LUAD) is the most common pathological subtype of lung cancer and surgery is its most common treatment. The dysregulated expression of DNA repair genes is found in a variety of cancers and has been shown to affect the origin and progression of these diseases. However, the function of DNA repair genes in surgically-treated LUAD is unclear.

Methods. We sought to determine the association between the signature of DNA repair genes for patients with surgical LUAD and their overall prognosis. We obtained gene expression data and corresponding clinical information of LUAD from The Cancer Genome Atlas (TCGA) database. The differently expressed DNA repair genes of surgically-treated LUAD and normal tissues were identified using the Wilcoxon rank-sum test. We used uni- and multivariate Cox regression analyses to shrink the aberrantly expressed genes, which were then used to construct the prognostic signature and the risk score formula associated with the independent prognosis of surgically-treated LUAD. We used Kaplan-Meier and Cox hazard ratio analyses to confirm the diagnostic and prognostic roles. Two validation sets (GSE31210 and GSE37745) were downloaded from the Gene Expression Omnibus (GEO) and were used to externally verify the prognostic value of the signature. OSluca online database verifies the hazard ratio for the DNA repair genes by which the signature was constructed. We investigated the correlation between the signature of the DNA repair genes and the clinical parameters. The potential molecular mechanisms and pathways of the prognostic signature were explored using Gene Set Enrichment Analysis (GSEA).

Results. We determined the prognostic signature based on 6 DNA repair genes (PLK1, FOXM1, PTTG1, CCNO, HIST3H2A, and BLM) and calculated the risk score based on this formula.

Patients with surgically-treated LUAD were divided into high-risk and low-risk groups according to the median risk score. The high-risk group showed poorer overall survival than the 
40 low-risk group; the signature was used as an independent prognostic indicator and had a greater 41 prognostic value in surgically-treated LUAD. The prognostic value was replicated in GSE31210 42 and GSE37745. OSluca online database analysis shows that 6 DNA repair genes were associated 43 with poor prognosis in most lung cancer datasets. The prognostic signature risk score correlated 44 with the pathological stage and smoking status in surgically-treated LUAD. The GSEA of the 45 risk signature in high-risk patients showed pathways associated with the cell cycle, oocyte 46 meiosis, mismatch repair, homologous recombination, and nucleotide excision repair.

47 Conclusions. A 6-DNA repair gene signature was determined using TCGA data mining and 48 GEO data verification. The gene signature may serve as a novel prognostic biomarker and 49 therapeutic target for surgically-treated LUAD.

50

51

52

53

54

55

56

57

58

59

60

61

62

63

64

65

66

67

68

69

70

71

72

73

74

75

76

77

78

\section{Introduction}

Lung cancer has the highest morbidity and mortality of cancers worldwide (Siegel et al. 2020).

Lung adenocarcinoma (LUAD) is the most common pathological subtype of lung cancer, accounting for approximately $40 \%$ of all cases (Denisenko et al. 2018). Surgery is the most common treatment for LUAD (Hirsch et al. 2017) although there is a high rate of relapse with $30-40 \%$ of patients dying from stage I surgically-treated LUAD (Oskarsdottir et al. 2016; Westaway et al. 2013). The 5-year overall survival (OS) rate decreases to $36 \%$ for those with stage III--IV surgically-treated LUAD (Mansuet-Lupo et al. 2014). The patients with surgically resected LUAD had varied prognoses, but patients with partially surgically-resected cancers had poor prognoses, regardless of whether the patients were early-LUAD treated with surgery alone or advanced-LUAD undergoing aggressive postoperative therapy (including platinum-based chemotherapy and radiation therapy). The biological mechanism of these differences is not fully understood (De Miguel-Perez et al. 2019). It is important to determine the prognoses of surgically-treated LUAD patients to provide specific treatments for improved OS.

Previous studies have shown that DNA repair systems play an important role in cancer(Gavande et al. 2016; Jeggo et al. 2016; Mei et al. 2019; Tessoulin et al. 2018). Five major DNA repair pathways are vital to maintaining the genetic stability of cells (Chatterjee \& Walker 2017): base excision repair (BER), nucleotide excision repair (NER), mismatch repair (MMR), homologous recombination (HR) and non-homologous end joining (NHEJ), two specific lesion repair pathways (direct chemical reversal and interstrand crosslink (ICL) repair) are important supplements for DNA damage repair. DNA repair genes play an essential role in tumorigenesis, tumor progression, and response to therapy(Lima et al. 2019). Efforts are being made to identify DNA damage repair genes as therapeutic targets and to develop agents against these targets. For example, poly ADP-ribose polymerase 1 (PARP1) is involved in multiple DNA repair pathways to maintain genomic stability so PARP1 inhibitors have been approved to treat ovarian cancer, breast cancer, and other DNA repair-deficient tumors (Ray Chaudhuri \& Nussenzweig 2017). LUAD exists in multiple DNA repair gene mutations but no studies have been conducted on DNA damage repair pathways in LUAD to date. We studied surgically-resected LUAD and the prognostic and predictive significance of the expression of DNA repair genes for this cancer. 
79 We constructed a multi-gene signature suitable for clinical application in surgical LUAD

80 patients, based on multi-gene signatures that indicate a poor prognosis in high-risk populations of

81 specific tumor patients. This approach increases our knowledge of tumor cell generation, growth,

82 and metastasis, and creates new avenues for targeted therapy. We obtained 201 surgically-treated

83 LUAD cases from The Cancer Genome Atlas (TCGA) database and extracted and identified key

84 mRNAs associated with DNA repair and established a 6-gene risk signature that could accurately

85 predict the prognosis of these specific patients.

\section{Materials \& Methods}

87 Data source and preprocessing

88 We extracted the gene expression profiles and corresponding clinical information of patients

89

90

91

92

93

94

95

96

97

98

99

100

101

102

103

104

105

106

107

108

109

110

111

112

113

114

115

116

117

118

119

120

121

with LUAD from the TCGA database (https://portal.gdc.cancer.gov/). We obtained the gene expression profiles of 59 normal samples and screened 535 LUAD samples for performance status, OS, survival state, and gene expression profiles. We collected data from 201 surgicallyresected LUAD cases. The gene mRNA expression data were normalized using the Transcripts Per Kilobase of exon model per Million mapped reads (TPM) method (Li et al. 2010). Clinical information including gender, age, pathological stage and TNM stage were included in this research. The Gene Expression Omnibus (GEO) database (https://www.ncbi.nlm.nih.gov/geo/) for microarray-based expression data was used to obtain data from patients with surgicallyresected LUAD, and that datasets to be considered must be containing more than 100 cases with information of OS and living status. The expression data of the GSE31210 set were normalized by the MAS5 algorithm and transformed with $\log 2$. The expression data of the GSE37745 set were normalized by the Robust Multi-Array Average (RMA) method and transformed with log2. 226 cases from GSE31210 (validation set 1) and 106 cases from GSE37745 (validation set 2) were screened with gene expression data and relevant clinical information. The OSluca online database (http://bioinfo.henu.edu.cn/LUCA/LUCAList.jsp) contains multiple lung cancer datasets(Yan et al. 2020), which can perform hazard ratio analysis on screened prognostic genes related to DNA repair. TCGA data were applied as a training set. GEO data sets were applied as validation sets. OS was defined as the date of the first treatment to the date of death of any cause or to the time of the last follow-up. The clinical characteristics are summarized in Table 1.

\section{Bioinformatic analysis}

Differential expression genes (DEGs) were filtered according to the adjusted $p$-value $<0.05$, $\mid \log 2$ fold change $\mid>1.5$ in the surgically-treated LUAD and normal samples of the training set. DNA repair genes were further screened from DEGs. Univariate Cox regression analysis evaluated the DNA repair genes significantly related to OS according to $p$-value $<0.05$. Multivariate Cox regression analysis was used for further screening. DNA repair genes are executors in the extremely complicated processes of DNA damage repairing which remedies specific types of DNA damage through specific pathways. We obtained a prognostic signature of 6 DNA repair genes and a corresponding prognostic risk score formula based on a linear combination of corresponding multivariate Cox regression coefficients. The formula for the risk score was: expression level of gene $1 \times \mathrm{K} 1+$ expression level of gene $2 \times \mathrm{K} 2+\cdots+$ expression of level gene $\mathrm{n} \times \mathrm{Kn}$. The risk score for every surgically-treated LUAD patient was calculated using this formula and the population was categorized into high-risk and low-risk groups according to the median risk score value. We used gene set enrichment analysis (GSEA) (https://www.gsea-

PeerJ reviewing PDF | (2020:05:48835:2:1:NEW 29 Oct 2020) 
122

123

124

125

126

127

128

129

130

131

132

133

134

135

136

137

138

139

140

141

142

143

144

145

146

147

148

149

150

151

152

153

154

155

156

157

158

159

160

161

162

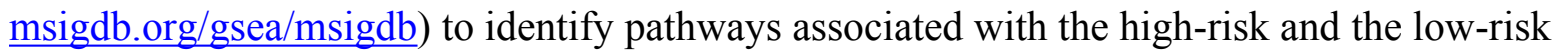
groups of DNA repair gene signatures. The Kyoto Encyclopedia of Genes and Genomes (KEGG) gene sets were downloaded from the MSigDB database. Enrichment false discovery rates (FDR) were based on 1,000 permutations. The high-risk score vs. low-risk score and FDR $<0.07$ were considered to be statistically significant.

\section{Statistical analysis}

The gene expression differences between the surgically-treated LUAD group and the normal group were compared using the unpaired two-tailed student's t-test. We evaluated the prognostic value of the risk score and clinicopathological features in patients with surgically-treated LUAD using univariate and multivariate Cox analysis. The Kaplan-Meier method was used to analyze the correlation between the risk score and OS. The survival curves were compared using a logrank test. All statistical analyses were performed using R 3.6.3 (https://www.r-project.org/) and SPSS 20.0.

\section{Results}

\section{Identification of DNA repair-related prognostic genes}

Study was conducted in accordance of the flow chart (Fig. 1). LUAD gene expression data and clinical records were downloaded from TCGA database. We screened DEGs from surgicallytreated LUAD and normal samples of the training dataset. In accordance with common senses and definition, we collected DNA repair-related gene sets from the MSigDB database and literature records (Table S1)(Pearl et al. 2015), and further selected from the DEGs to identify the DNA repair-related prognostic gene signature for surgically-treated LUAD. We acquired 68 genes related to DNA repair (Fig. 2A). Among them, the expression levels of 4 genes in surgical LUAD were lower than normal samples, while the other genes were the opposite. (Fig.2B, Fig.2C). These genes were used in Univariate Cox regression analysis and resulted in 23 genes significantly correlated with OS (Fig. 2D). The 23 genes were screened by multivariate Cox regression analysis, resulting in a total of 6 genes, namely PLK1, FOXM1, PTTG1, CCNO, HIST3H2A and BLM. A prognostic signature was constructed (Table 2) based on the 6 DNA repair-related prognostic genes.

\section{The performance of DNA repair-related prognostic gene signature}

The risk score for surgically-treated LUAD patients was calculated based on the gene mRNA level and corresponding coefficients using the formula: Risk score $=(0.555713) \times$ PLK1 expression value $+(-0.5696) \times$ FOXM1 expression value $+(0.291471) \times$ PTTG1 expression value $+(-0.30485) \times$ CCNO expression value $+(-0.17162) \times$ HIST3H2A expression value + $(0.442472) \times$ BLM expression value. The patients were divided into high-risk and low-risk groups based on the median risk score. The patients' risk score distribution and OS are shown in Fig. 3A and 3B. The expression profile distribution of 6 DNA repair-related genes is shown in Fig. 3C. A Kaplan-Meier curve showed poor prognoses for the high-risk group (log-rank test pvalue $<0.0001$; Fig. 3D). We used univariate and multivariate Cox hazard ratio analysis to further assess the performance of our signature risk score in surgically-treated LUAD patients based on other common clinicopathological features. Univariate Cox regression analysis showed that tumor stage and $\mathrm{N}$ classification were significantly correlated with survival. $\mathrm{N}$ classification 
163 was significantly correlated with survival in multivariate Cox regression analysis. Remarkably,

164

165

166

167

168

169

170

171

172

173

174

175

176

177

178

179

180

181

182

183

184

185

186

187

188

189

190

191

192

193

194

195

196

197

198

199

200

201

202

univariate and multivariate Cox analysis showed that the risk score was more significantly correlated with survival, giving it a greater prognostic value (Figs. 3E and 3F). These results indicated that the 6-gene signature risk score could reliably predict the survival of surgical LUAD patients.

\section{Validation of the 6-gene signature for survival prediction}

Gene expression data and clinical information of LUAD patients with surgical records and survival times were selected from the GEO database to further prove the accuracy of our gene signature. We obtained the GSE31210 and GSE37745 data sets. The GSE31210 expression data were standardization and distributed as shown in Fig. 4A and 4B. The GSE37745 set contained data from pathological samples of squamous cell lung carcinoma, lung adenocarcinoma, and large-cell carcinoma. We screened surgically-treated LUAD samples; their standardization and distribution is shown in Fig. 4C and 4D. The DNA repair-related signature and risk score formulas were applied to verify the two validation sets and the Kaplan-Meier curve showed that the high-risk group had poor OS when compared with the low-risk group (Fig. 4E pvalue $=0.008$, Fig. $4 \mathrm{~F} p$-value $=0.003$ ). Apart from that, multivariate Cox hazard ratio regression analysis in the validation sets GSE31210 and GSE37745 had also been conducted. According to the results, risk score can be considered as an independent risk factor (Fig. 4G HR=1.523, pvalue $=0.023$ ) in the GSE37745 set, but since only clinical stage I and II patients are included in the GSE31210 set differing from the training set TCGA which contains clinical I, II, III, IV stages, it could be insufficient regarding risk score as an independent risk factor after the inclusion of stage staging (Fig. $4 \mathrm{H} \mathrm{HR}=1.083$, $\mathrm{p}$-value $=0.072$ ). The $6 \mathrm{DNA}$ repair-related genes of the signature were analyzed in the OSluca online database for forest plot prognostic analysis. The results showed that these 6 genes were associated with poor prognosis in most lung cancer data sets (Fig.5, HR>1). The above results indicated that the signature had greater external validity and reliability.

\section{Relationship between 6-gene signature risk score and clinical parameters}

We studied the correlation between the 6-gene signature risk score and the clinical parameters under the training and validation sets. The sets were divided into two subgroups that included age, gender, pathological stage, TNM stage, and smoking status. The stratification results are shown in Table 1. In the training set, parameters such as age, gender, TNM stage did not affect risk score except for the correlation between pathological stage and risk score (Fig. 6A) (p-value $=0.022$ ). In the GSE31210 set, the pathological stage and smoking status were correlated with the risk score, but age and gender were not related to the risk score (Fig. 6B) (p-value $=0.0004, p$ value $=0.027$, respectively). In the GSE37745 set, age, gender and pathological stage were not related to the risk score (Fig. 6C).

\section{The biological processes and pathways associated with the 6-gene signature}

The population was divided into a high-risk group and a low-risk group based on the risk score of DNA repair gene expression and the prognosis between the two groups was significantly different. We examined the whole gene expression profile in TCGA data using GSEA analysis to 
203 reveal the potential mechanism of the 6-gene signature, which indicated that the main enrichment

204 pathways were the cell cycle, oocyte meiosis, DNA replication, mismatch repair, homologous

205 recombination and nucleotide excision repair pathways. Our results suggested that the poor

206 prognosis of patients with surgically-resected LUAD was related to the DNA damage repair

207 pathway (Fig. 7).

\section{Discussion}

209 Lung cancer has the highest mortality rate of malignant tumors and adenocarcinoma is the most

210 common histological subtype (Network 2014). Video-assisted thoracoscopic surgical lobectomy

211 for early-stage NSCLC and robotic lobectomy for advanced NSCLC is the primary surgical

212 treatment for LUAD (Cerfolio et al. 2018; Chen et al. 2013; Veronesi et al. 2018).

213 Advancements in imaging techniques, diagnostic methods, surgical skills, and anesthesia

214 management have led to an increase in the number of LUAD patients who were evaluated for

215 and underwent surgery (Ucvet et al. 2020). Surgical treatment of patients with LUAD can extend

216 survival time but there may be limited benefits to the partial surgical treatment of LUAD patients

217 because of local recurrence and distant metastasis. Therefore, effective prognostic and

218 therapeutic strategies to improve the survival rate of surgical LUAD are being actively explored.

219 The development of high-throughput sequencing technology has improved the identification of

220 potential biomarkers for tumor progression and prognosis. For example, Xu et al. (2020)

221 confirmed that flamingo subfamily Cadherin EGF LAG seven-pass G-type receptor 2 (CELSR2)

222 is significantly overexpressed in hepatocellular carcinoma and may serve as a novel prognostic

223 biomarker. Feng et al. (2020) used the Cox proportional hazards model to confirm that MHC

224 class I chain-related B (MICB) expression was significantly associated with clinical parameters

225 in colorectal cancer (CRC) and high MICB expression was an independent protective factor for

226 OS. However, the predictive power of such biomarkers is limited in context-specific cancers

227 because it is influenced by many factors, including transcription modification, translation

228 modification, and temporal and spatial heterogeneity. Thus, a signature based on multi-gene

229 cooperation can improve the predictive ability of each gene, resulting in a more reliable and

230 robust predictive ability versus a single biomarker (Ashley 2015; Jiang et al. 2020; Wolfe et al.

231 2020; Zhu et al. 2016). DNA damage repair genes are needed to maintain cell homeostasis and

232 are extensively involved in cell replication, chromatin modification, DNA repair, checkpoint

233 signaling, reactive oxygen species, metabolism, senescence, and apoptosis cellular mechanisms

234 (Brinkman et al. 2020; Csiszar et al. 2019; Gonzalo \& Coll-Bonfill 2019; Lee et al. 2018; Royce

235 et al. 2019; Ungvari et al. 2019). Genetic mutations can effectively induce tumorigenesis and

236 progression (Pearl et al. 2015). Targeted inhibition of the function of specific genes in DNA

237 damage repair response has potential for clinical applications and the development of targeted

238 drugs (Li et al. 2019; Nickoloff et al. 2017; O'Connor 2015; Tarantini et al. 2019). We used

239 DNA repair genes to construct and validate a prognostic signature that successfully predicts the

240 survival time of surgically-treated LUAD patients.

241 We filtered the data downloaded from the TCGA database to obtain gene expression profiles and

242 clinical data of patients with surgically-treated LUAD. The standardized DNA repair gene DEGs

243 were screened for OS-related univariate and multivariate Cox regression analysis and the DNA

244 repair-related prognosis signature and risk score calculation formulas were obtained. We divided

245 the surgically-treated LUAD patients into high- and low-risk groups according to the calculated

246 formula. Kaplan-Meier survival analysis showed that the high-risk group had a poor prognosis

PeerJ reviewing PDF | (2020:05:48835:2:1:NEW 29 Oct 2020) 
247 and Cox analysis showed that the risk score could be used as an independent prognostic factor, 248 verifying the predictive effect of the signature. The GSE31210 and GSE377745 sets downloaded 249 from GEO were used to perform survival analysis and the results were consistent with those 250 from the Kaplan-Meier and Cox analyses. The DNA repair-related gene signature was shown to 251 be highly reliable. GSEA analysis suggested that the poor prognosis of patients with surgically252 resected LUAD was related to the DNA damage repair pathway, according to p-value $<0.05$ and 253 FDR $<0.07$.

254 We obtained 6 DNA repair genes making up the prognostic signature. Some of the 6 genes 255 (PLK1, FOXM1, PTTG1, CCNO, HIST3H2A and BLM) were associated with tumorigenesis. 256 PLK1 is a member of the Polo-like kinases (PLKs) family of serine/threonine protein kinases and 257 plays an essential role in many stages of mitosis. The expression of PLK1 is cell cycle-dependent 258 259 260 261

262 263 and its expression peaks in the M phase of a normal cell. PLK1 is highly expressed in many human cancers and its overexpression is associated with a poor prognosis. Previous studies have shown that the PLK1 protein interacts with multiple tumor suppressors to cause tumorigenesis and that targeting the protein can improve the sensitivity of tumors to chemoradiotherapy. The target PLK1 inhibitor has shown considerable promise in clinical studies and is being tested in clinical trials (Gutteridge et al. 2016; Liu et al. 2017). FOXM1 is a proliferation specific

265

266

267

268

269

270

271

272

273

274

275

276

277

278

279

280

281

282

283

284

285 transcriptional modulator and is involved in the cell cycle, mitotic spindle integrity, angiogenesis, metastasis, apoptosis, and DNA damage repair pathway. This protein dysfunction contributes to tumorigenesis (Nandi et al. 2018). A meta-analysis reported that elevated FOXM1protein expression was significantly associated with poor survival in most solid tumors. This suggests that FOXM1 is a potential biomarker for predicting prognoses in solid human tumors (Li et al. 2017). PTTG1 has been identified as a critical signature gene associated with tumor metastasis. It is overexpressed in a variety of endocrine-related tumors especially those of the pituitary, breast, and testis, as well as in nonendocrine-related cancers involving the pulmonary and gastrointestinal systems (Vlotides et al. 2007). Studies have shown that PTTG1 is highly expressed in breast cancer and PTTG1 may increase breast cancer cell growth through the nuclear exclusion of p27 (Xiea \& Wangb 2016). BLM is a member of the RecQ helicases, and can unwind forked dsDNA and anneal ssDNA, and play critical roles in DNA repair, recombination, replication, and transcription. The mutations of BLM cause Bloom syndrome (Croteau et al. 2014). The small molecule inhibitor of BLM, ML216, exhibits cell-based activity and can induce sister chromatid exchanges, enhance the toxicity of aphidicolin, and exert antiproliferative activity in cells expressing BLM (Nguyen et al. 2013). CCNO and HIST3H2A were less prevalent in the high-risk group and there are few reports about the two genes. CCNO is part of the cyclin family and is an essential regulator of endogenous apoptosis and participates in DNA damage repair (Krokan \& Bjoras 2013). HIST3H2A is a replication-dependent histone H2A gene located in the HIST3 cluster on chromosome 1 in the human genome sequence. We integrated the 6 DNA repair genes into a signature and found that patients in the high-risk group were associated with worse survival, which confirms the predictive value of the DNA repair 
286

287

288

289

290

291

292

293

294

295

296

297

298

299

300

301

302

303

304

305

306

307

308

309

310

311

312

313

314

315

316

317

318

319

320

321

322

323

324

325

326

327

gene signature in surgically-treated LUAD. The development of drugs against these target genes may be used as a potential treatment in surgically-treated LUAD.

We filtered out patients with combined radiotherapy, chemotherapy, neoadjuvant therapy and postoperative adjuvant therapy within the pre-set framework. In TCGA, due to the small number of LUAD cases with surgical treatment combined with radiotherapy, neoadjuvant treatment or adjuvant treatment, we did not conduct further analysis. 69 cases were found with chemotherapy and the survival rate of low-risk group was higher, but no significant difference was obtained $(\mathrm{p}=0.17)$ due to the small size of the sample, follow-up needs to expand the case for verification. Tumor markers, as a class of substances synthesized and released by tumor cells, or increased as the body reacting to tumor cells, are mainly applied for auxiliary diagnosis, prognostic judgment, curative effect observation and guiding follow-up treatment in current clinical practice. Although being convenient, highly-efficient, repeatably-detected and of low price, they are still limited by its relatively poor specificity and sensitivity. As specified for patients with LUAD after surgery, our multi-gene signature is supposed to have better specificity and the efficacy of radiotherapy can be predicted based on the expression of DNA repair-related genes for those with postoperative radiotherapy combined. Disadvantages mainly include the need for clinical sample sequencing which could be difficult to obtain and high sequencing cost.

We use data mining and bioinformatics analysis on the TCGA, GEO and OSluca databases to construct a DNA repair-related gene prognosis signature. Since the number of enrolled cases is relatively small, more cases should be recruited to expand the study. In addition, we need more clinical practice to further verify our conclusions.

\section{Conclusions}

We used data from surgically-treated LUAD to identify DNA repair-related DEGs and constructed and validated a 6-gene signature for predicting the outcomes of surgically-treated LUAD. Further study of these DNA repair-related genes showed that the prognosis-risk signature of DNA repair-related genes can be used as potential predictive markers and therapeutic targets for the surgical treatment of LUAD.

\section{Acknowledgements}

Thanks to all the researchers and staff working for The Cancer Genome Atlas database and Gene Expression Omnibus Database.

\section{References}

Ashley EA. 2015. The precision medicine initiative: a new national effort. Jama 313:2119-2120. 10.1001/jama.2015.3595

Brinkman JA, Liu Y, and Kron SJ. 2020. Small-molecule drug repurposing to target DNA damage repair and response pathways. Seminars in Cancer Biology. 10.1016/j.semcancer.2020.02.013

Cerfolio RJ, Ghanim AF, Dylewski M, Veronesi G, Spaggiari L, and Park BJ. 2018. The longterm survival of robotic lobectomy for non-small cell lung cancer: A multi-institutional study. J Thorac Cardiovasc Surg 155:778-786. 10.1016/j.jtcvs.2017.09.016

Chatterjee N, and Walker GC. 2017. Mechanisms of DNA damage, repair, and mutagenesis. Environ Mol Mutagen 58:235-263. 10.1002/em.22087 
328

329

330

331

332

333

334

335

336

337

338

339

340

341

342

343

344

345

346

347

348

349

350

351

352

353

354

355

356

357

358

359

360

361

362

363

364

365

366

367

368

369

370

371

372

373

374

375

376

377

Chen FF, Zhang D, Wang YL, and Xiong B. 2013. Video-assisted thoracoscopic surgery lobectomy versus open lobectomy in patients with clinical stage non-small cell lung cancer: a meta-analysis. Eur J Surg Oncol 39:957-963. 10.1016/j.ejso.2013.06.016

Croteau DL, Popuri V, Opresko PL, and Bohr VA. 2014. Human RecQ helicases in DNA repair, recombination, and replication. Annu Rev Biochem 83:519-552. 10.1146/annurevbiochem-060713-035428

Csiszar A, Balasubramanian P, Tarantini S, Yabluchanskiy A, Zhang XA, Springo Z, Benbrook D, Sonntag WE, and Ungvari Z. 2019. Chemically induced carcinogenesis in rodent models of aging: assessing organismal resilience to genotoxic stressors in geroscience research. Geroscience 41:209-227. 10.1007/s11357-019-00064-4

De Miguel-Perez D, Bayarri-Lara Cl, Ortega FG, Russo A, Moyano Rodriguez MJ, AlvarezCubero MJ, Maza Serrano E, Lorente JA, Rolfo C, and Serrano MJ. 2019. Post-Surgery Circulating Tumor Cells and AXL Overexpression as New Poor Prognostic Biomarkers in Resected Lung Adenocarcinoma. Cancers (Basel) 11. 10.3390/cancers11111750

Denisenko TV, Budkevich IN, and Zhivotovsky B. 2018. Cell death-based treatment of lung adenocarcinoma. Cell Death Dis 9:117. 10.1038/s41419-017-0063-y

Gavande NS, VanderVere-Carozza PS, Hinshaw HD, Jalal SI, Sears CR, Pawelczak KS, and Turchi JJ. 2016. DNA repair targeted therapy: The past or future of cancer treatment? Pharmacol Ther 160:65-83. 10.1016/j.pharmthera.2016.02.003

Gonzalo S, and Coll-Bonfill N. 2019. Genomic instability and innate immune responses to selfDNA in progeria. Geroscience 41:255-266. 10.1007/s11357-019-00082-2

Gutteridge REA, Ndiaye MA, Liu X, and Ahmad N. 2016. Plk1 Inhibitors in Cancer Therapy: From Laboratory to Clinics. Molecular Cancer Therapeutics 15:1427-1435. 10.1158/1535-7163.Mct-15-0897

Hirsch FR, Scagliotti GV, Mulshine JL, Kwon R, Curran WJ, Wu Y-L, and Paz-Ares L. 2017. Lung cancer: current therapies and new targeted treatments. The Lancet 389:299-311. 10.1016/s0140-6736(16)30958-8

Jeggo PA, Pearl LH, and Carr AM. 2016. DNA repair, genome stability and cancer: a historical perspective. Nat Rev Cancer 16:35-42. 10.1038/nrc.2015.4

Jiang W, Zhu D, Wang C, and Zhu Y. 2020. An immune relevant signature for predicting prognoses and immunotherapeutic responses in patients with muscle - invasive bladder cancer (MIBC). Cancer Medicine 9:2774-2790. 10.1002/cam4.2942

Krokan HE, and Bjoras M. 2013. Base excision repair. Cold Spring Harb Perspect Biol 5:a012583. 10.1101/cshperspect.a012583

Lee HJ, Feliers D, Barnes JL, Oh S, Choudhury GG, Diaz V, Galvan V, Strong R, Nelson J, Salmon A, Kevil CG, and Kasinath BS. 2018. Hydrogen sulfide ameliorates agingassociated changes in the kidney. Geroscience 40:163-176. 10.1007/s11357-018-0018$y$

Li A, Yi M, Qin S, Chu Q, Luo S, and Wu K. 2019. Prospects for combining immune checkpoint blockade with PARP inhibition. J Hematol Oncol 12:98. 10.1186/s13045-019-0784-8

Li B, Ruotti V, Stewart RM, Thomson JA, and Dewey CN. 2010. RNA-Seq gene expression estimation with read mapping uncertainty. Bioinformatics 26:493-500.

10.1093/bioinformatics/btp692

Li L, Wu D, Yu Q, Li L, and Wu P. 2017. Prognostic value of FOXM1 in solid tumors: a systematic review and meta-analysis. Oncotarget 8:32298-32308. 10.18632/oncotarget.15764

Lima ZS, Ghadamzadeh M, Arashloo FT, Amjad G, Ebadi MR, and Younesi L. 2019. Recent advances of therapeutic targets based on the molecular signature in breast cancer: genetic mutations and implications for current treatment paradigms. $J$ Hematol Oncol 12:38. 10.1186/s13045-019-0725-6

Peer) reviewing PDF | (2020:05:48835:2:1:NEW 29 Oct 2020) 
378

379

380

381

382

383

384

385

386

387

388

389

390

391

392

393

394

395

396

397

398

399

400

401

402

403

404

405

406

407

408

409

410

411

412

413

414

415

416

417

418

419

420

421

422

423

424

425

426

427

428

Liu Z, Sun Q, and Wang X. 2017. PLK1, A Potential Target for Cancer Therapy. Translational Oncology 10:22-32. 10.1016/j.tranon.2016.10.003

Mansuet-Lupo A, Bobbio A, Blons H, Becht E, Ouakrim H, Didelot A, Charpentier M-C, Bain S, Marmey B, Bonjour P, Biton J, Cremer I, Dieu-Nosjean M-C, Sautès-Fridman C, Régnard J-F, Laurent-Puig P, Alifano M, and Damotte D. 2014. The New Histologic Classification of Lung Primary Adenocarcinoma Subtypes Is a Reliable Prognostic Marker and Identifies Tumors With Different Mutation Status. Chest 146:633-643. 10.1378/chest.13-2499

Mei L, Zhang J, He K, and Zhang J. 2019. Ataxia telangiectasia and Rad3-related inhibitors and cancer therapy: where we stand. J Hematol Oncol 12:43. 10.1186/s13045-019-0733-6

Nandi D, Cheema PS, Jaiswal N, and Nag A. 2018. FoxM1: Repurposing an oncogene as a biomarker. Seminars in Cancer Biology 52:74-84. 10.1016/j.semcancer.2017.08.009

Network TCGAR. 2014. Comprehensive molecular profiling of lung adenocarcinoma. Nature 511:543-550. 10.1038/nature13385

Nguyen GH, Dexheimer TS, Rosenthal AS, Chu WK, Singh DK, Mosedale G, Bachrati CZ, Schultz L, Sakurai M, Savitsky P, Abu M, McHugh PJ, Bohr VA, Harris CC, Jadhav A, Gileadi O, Maloney DJ, Simeonov A, and Hickson ID. 2013. A small molecule inhibitor of the BLM helicase modulates chromosome stability in human cells. Chem Biol 20:55-62. 10.1016/j.chembiol.2012.10.016

Nickoloff JA, Jones D, Lee SH, Williamson EA, and Hromas R. 2017. Drugging the Cancers Addicted to DNA Repair. J Natl Cancer Inst 109. 10.1093/jnci/djx059

O'Connor MJ. 2015. Targeting the DNA Damage Response in Cancer. Mol Cell 60:547-560. 10.1016/j.molcel.2015.10.040

Oskarsdottir GN, Bjornsson J, Jonsson S, Isaksson HJ, and Gudbjartsson T. 2016. Primary adenocarcinoma of the lung - histological subtypes and outcome after surgery, using the IASLC/ATS/ERS classification of lung adenocarcinoma. Apmis 124:384-392. 10.1111/apm. 12522

Pearl LH, Schierz AC, Ward SE, Al-Lazikani B, and Pearl FM. 2015. Therapeutic opportunities within the DNA damage response. Nat Rev Cancer 15:166-180. 10.1038/nrc3891

Ray Chaudhuri A, and Nussenzweig A. 2017. The multifaceted roles of PARP1 in DNA repair and chromatin remodelling. Nat Rev Mol Cell Biol 18:610-621. 10.1038/nrm.2017.53

Royce GH, Brown-Borg HM, and Deepa SS. 2019. The potential role of necroptosis in inflammaging and aging. Geroscience 41:795-811. 10.1007/s11357-019-00131-w

Siegel RL, Miller KD, and Jemal A. 2020. Cancer statistics, 2020. CA Cancer J Clin 70:7-30. 10.3322/caac. 21590

Tarantini S, Yabluchanskiy A, Csipo T, Fulop G, Kiss T, Balasubramanian P, DelFavero J, Ahire C, Ungvari A, Nyúl-Tóth Á, Farkas E, Benyo Z, Tóth A, Csiszar A, and Ungvari Z. 2019. Treatment with the poly(ADP-ribose) polymerase inhibitor PJ-34 improves cerebromicrovascular endothelial function, neurovascular coupling responses and cognitive performance in aged mice, supporting the NAD+ depletion hypothesis of neurovascular aging. Geroscience 41:533-542. 10.1007/s11357-019-00101-2

Tessoulin B, Moreau-Aubry A, Descamps G, Gomez-Bougie P, Maïga S, Gaignard A, Chiron D, Ménoret E, Le Gouill S, Moreau P, Amiot M, and Pellat-Deceunynck C. 2018. Wholeexon sequencing of human myeloma cell lines shows mutations related to myeloma patients at relapse with major hits in the DNA regulation and repair pathways. $J$ Hematol Oncol 11:137. 10.1186/s13045-018-0679-0

Ucvet A, Gursoy S, and Yazgan S. 2020. Changes in the Surgical Treatment Strategies for Nonsmall Cell Lung Cancer in the Past 20 Years: A Single-Center Experience. Turk Thorac J 21:8-13. 10.5152/TurkThoracJ.2019.180124

Ungvari Z, Tarantini S, Nyúl-Tóth Á, Kiss T, Yabluchanskiy A, Csipo T, Balasubramanian P, Lipecz A, Benyo Z, and Csiszar A. 2019. Nrf2 dysfunction and impaired cellular

Peer) reviewing PDF | (2020:05:48835:2:1:NEW 29 Oct 2020) 
429

430

431

432

433

434

435

436

437

438

439

440

441

442

443

444

445

446

447

448

449

450

451

452

453

454

455 resilience to oxidative stressors in the aged vasculature: from increased cellular senescence to the pathogenesis of age-related vascular diseases. Geroscience 41:727738. 10.1007/s11357-019-00107-w

Veronesi G, Park B, Cerfolio R, Dylewski M, Toker A, Fontaine JP, Hanna WC, Morenghi E, Novellis P, Velez-Cubian FO, Amaral MH, Dieci E, Alloisio M, and Toloza EM. 2018. Robotic resection of Stage III lung cancer: an international retrospective study†. European Journal of Cardio-Thoracic Surgery 54:912-919. 10.1093/ejcts/ezy166

Vlotides G, Eigler T, and Melmed S. 2007. Pituitary tumor-transforming gene: physiology and implications for tumorigenesis. Endocr Rev 28:165-186. 10.1210/er.2006-0042

Westaway DD, Toon CW, Farzin M, Sioson L, Watson N, Brady PW, Marshman D, Mathur MM, and Gill AJ. 2013. The International Association for the Study of Lung Cancer/American Thoracic Society/European Respiratory Society grading system has limited prognostic significance in advanced resected pulmonary adenocarcinoma. Pathology 45:553-558. 10.1097/PAT.0b013e32836532ae

Wolfe AR, Wald P, Webb A, Sebastian N, Walston S, Robb R, Chen W, Vedaie M, Dillhoff M, Frankel WL, Kwon W, Jang JY, and Williams TM. 2020. A microRNA-based signature predicts local-regional failure and overall survival after pancreatic cancer resection. Oncotarget 11:913-923. 10.18632/oncotarget.27496

Xiea Y, and Wangb R. 2016. Pttg1 Promotes Growth of Breast Cancer through P27 Nuclear Exclusion. Cell Physiol Biochem 38:393-400. 10.1159/000438660

Yan Z, Wang Q, Lu Z, Sun X, Song P, Dang Y, Xie L, Zhang L, Li Y, Zhu W, Xie T, Ma J, Zhang Y, and Guo X. 2020. OSluca: An Interactive Web Server to Evaluate Prognostic Biomarkers for Lung Cancer. Frontiers in Genetics 11. 10.3389/fgene. 2020.00420

Zhu X, Tian X, Yu C, Shen C, Yan T, Hong J, Wang Z, Fang JY, and Chen H. 2016. A long noncoding RNA signature to improve prognosis prediction of gastric cancer. Mol Cancer 15:60. 10.1186/s12943-016-0544-0 


\section{Table $\mathbf{1}$ (on next page)}

Clinic pathological characteristics of surgical LUAD patients from the training(TCGA) and validation sets(GSE31210, GSE37745) 


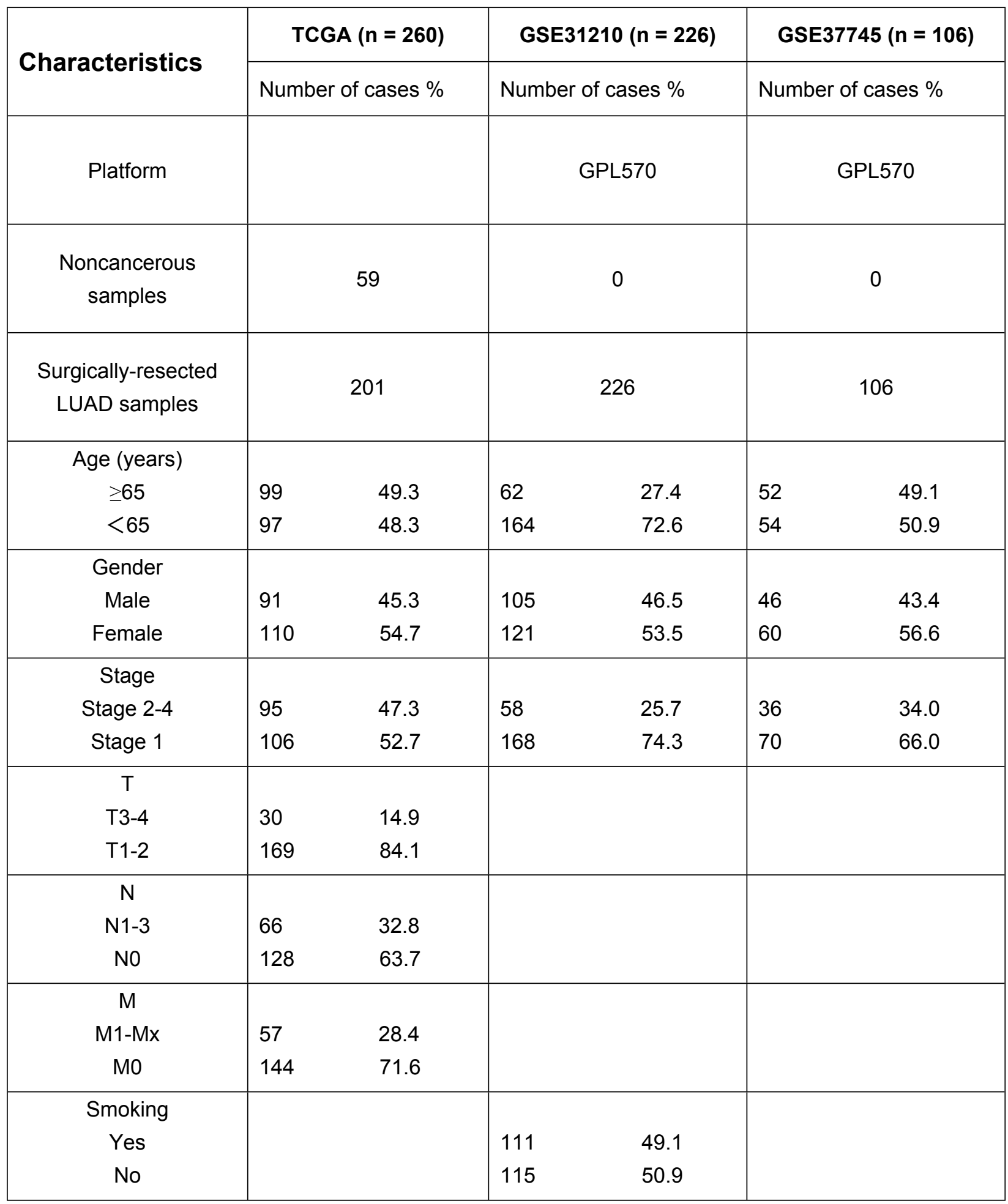

2 Notes: Abbreviations: LUAD, lung adenocarcinoma; T, tumor size; N, lymph node status; $M$, 3 metastasis. 
Table 2 (on next page)

The information of 6 prognostic mRNAs importantly associated with overall survival in patients with surgical LUAD 


\begin{tabular}{|c|c|c|c|c|c|}
\hline mRNA & Ensemble ID & Location & Coefficient & HR & P-value \\
\hline PLK1 & ENSG00000166851 & $\begin{array}{c}\text { Chr16:23,677,656- } \\
23,690,367\end{array}$ & 0.555713 & 1.743183 & 0.043687 \\
\hline FOXM1 & ENSG00000111206 & $\begin{array}{c}\text { Chr12: } 2,857,681- \\
2,877,155\end{array}$ & -0.5696 & 0.565754 & 0.024525 \\
\hline PTTG1 & ENSG00000164611 & $\begin{array}{c}\text { Chr5: } 160,421,855- \\
160,428,739\end{array}$ & 0.291471 & 1.338395 & 0.064214 \\
\hline CCNO & ENSG00000152669 & $\begin{array}{c}\text { Chr5: } 55,231,152- \\
55,233,608\end{array}$ & -0.30485 & 0.737234 & 0.004893 \\
\hline HIST3H2 & ENSG00000181218 & $\begin{array}{c}\text { Chr1: } 228,456,979- \\
228,457,873\end{array}$ & -0.17162 & 0.842298 & 0.030497 \\
\hline BLM & ENSG00000197299 & $\begin{array}{c}\text { Chr15: } 90,717,346- \\
90,816,166\end{array}$ & 0.442472 & 1.556551 & 0.119493 \\
\hline
\end{tabular}

2 Notes: Abbreviations: LUAD, lung adenocarcinoma; HR, hazard ratio; ID, identity 
Figure 1

Flow diagram of methods for developing and verifying the 6 DNA repair-related prognostic genes signature.

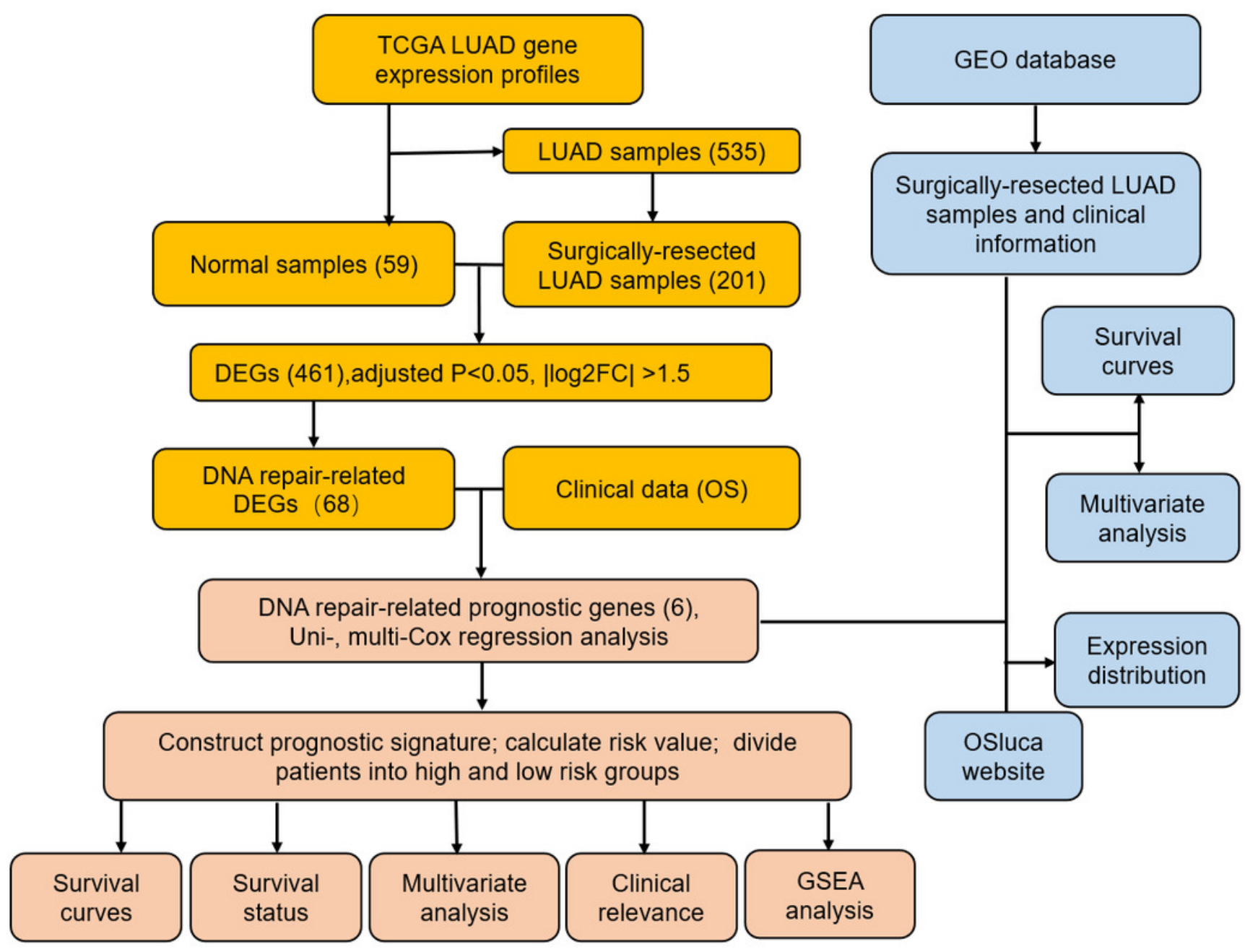




\section{Figure 2}

Identification of DNA repair-related prognostic genes.

(A) Volcano plot of DNA repair-related DEGs. $\mid \log 2$ (Fold Change) $\mid>1.5$ and FDR $<0.05$ were set as screening criteria. The green, red and black dots represented the down-, up-regulated DNA repair-related DEGs and genes that were not satisfied the screening criteria, respectively. (B) The heatmap of all 68 DNA repair-related DEGs distributions. (C) The Boxplot of all 68 DNA repair-related DEGs distributions. (D) The forest plot of 23 prognostic DNA repair-related DEGs screened out by univariate Cox regression. DEGs, Differentially expressed genes; N, Normal samples; T, Surgical lung adenocarcinoma. 
A

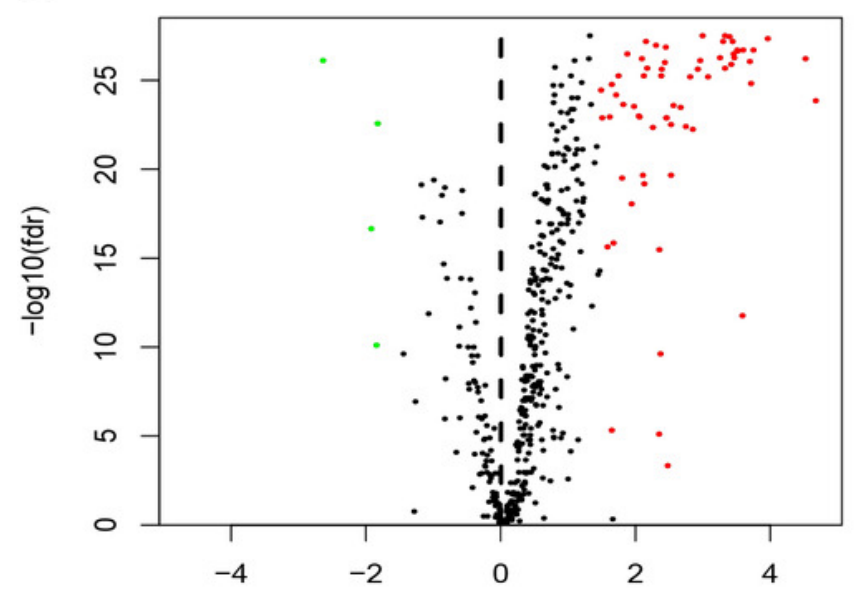

C

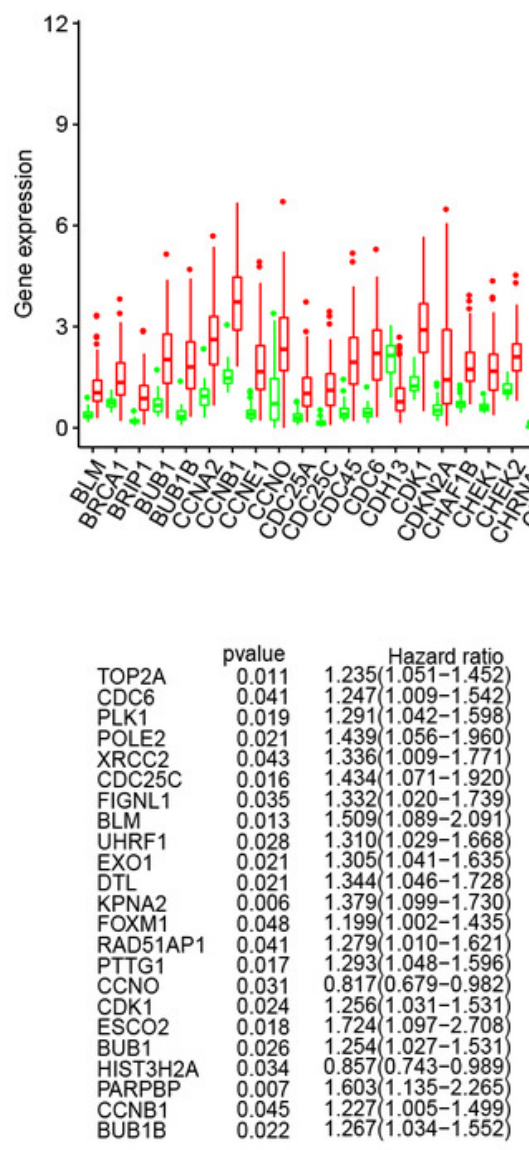

$\log \mathrm{FC}$
B

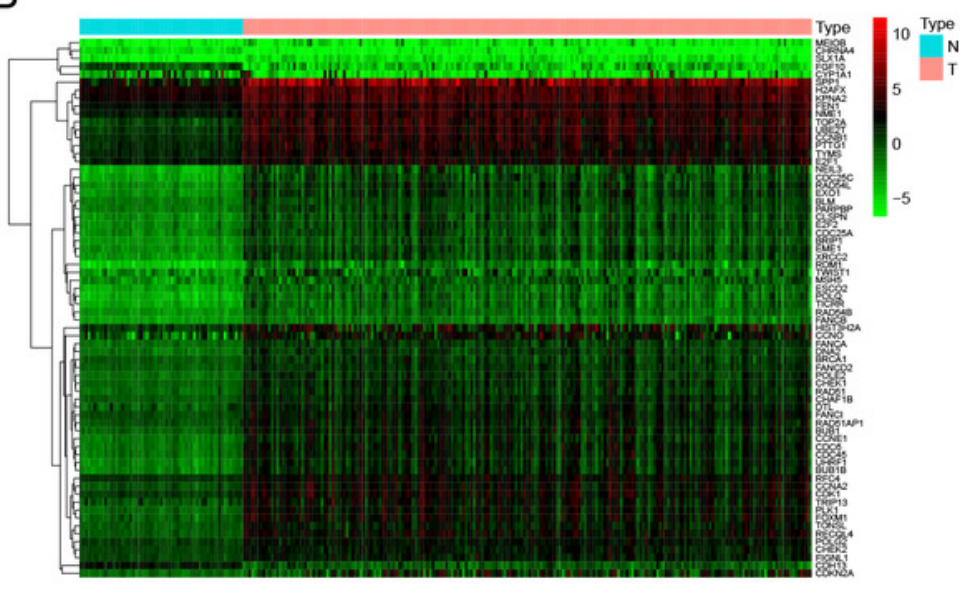

type 宁 $\mathrm{N}$ 宁

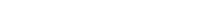




\section{Figure 3}

The performance of DNA repair-related prognostic gene signature.

(A) 6 prognostic DNA repair-related DEGs screened out by multivariate Cox regression to construct signature and calculate risk score, the distribution of risk score of each patient. (B) The distribution of survival status of each patient. (C) The heatmap of 6 DNA repair-related signature DEGs distributions in risk score groups. (D) Kaplan-Meier survival curves for overall survival in the DNA repair-related risk score groups. (E) Univariate Cox hazard ratio analysis shown the signature risk score and other clinicopathological features related to overall survival of surgical LUAD. (F) Multivariate Cox hazard ratio analysis shown the signature risk score and other clinicopathological features related to overall survival of surgical LUAD. High: high risk; Low: low risk; gender:0 (female); 1 (male); stage: 1, 2, 3, 4; T:1, 2, 3, 4;N:0, 1, 2, 3, $M: 0,1$. 
A

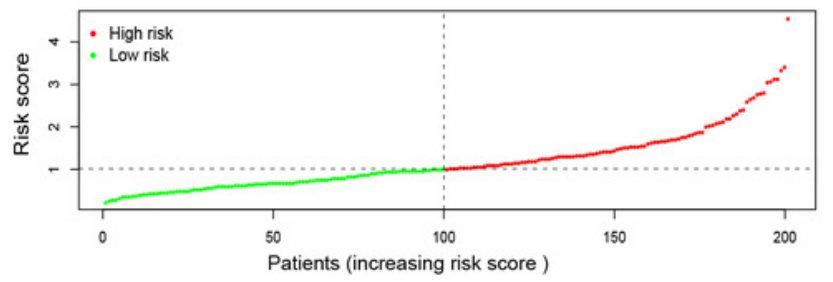

B

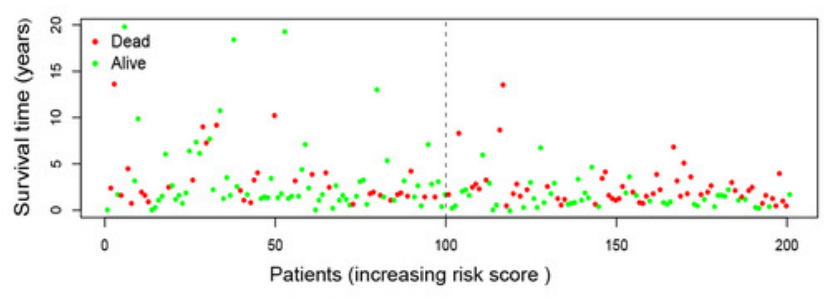

D

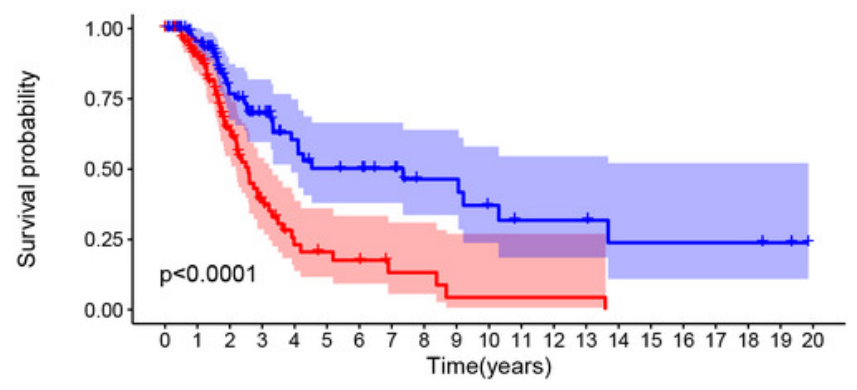

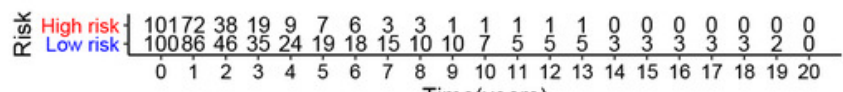

$\mathrm{F}$
C

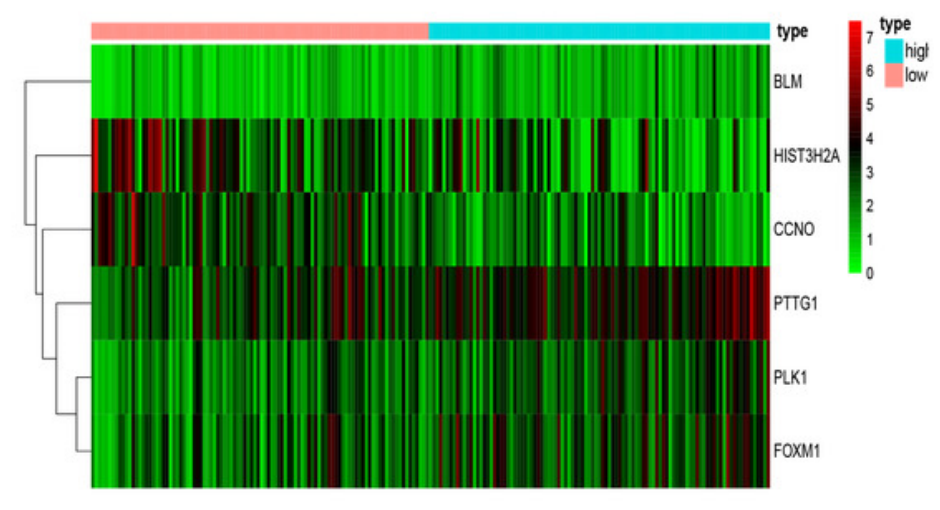

E

$\begin{array}{lrr} & \text { pvalue } & \text { Hazard ratio } \\ \text { age } & 0.223 & 1.014(0.992-1.037) \\ \text { gender } & 0.273 & 0.780(0.500-1.216) \\ \text { stage } & 0.002 & 1.406(1.138-1.736) \\ \text { T } & 0.217 & 1.191(0.902-1.573) \\ \mathrm{N} & 0.024 & 1.382(1.043-1.830) \\ \mathrm{M} & 0.097 & 1.864(0.893-3.888) \\ \text { riskScore } & <0.001 & 1.909(1.482-2.460)\end{array}$

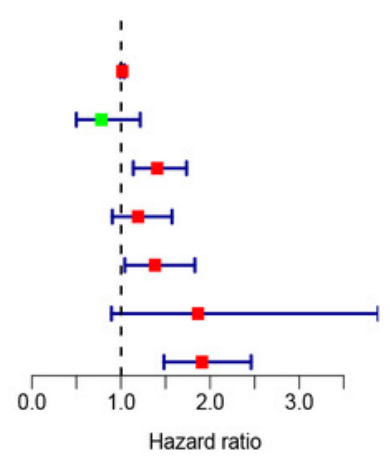

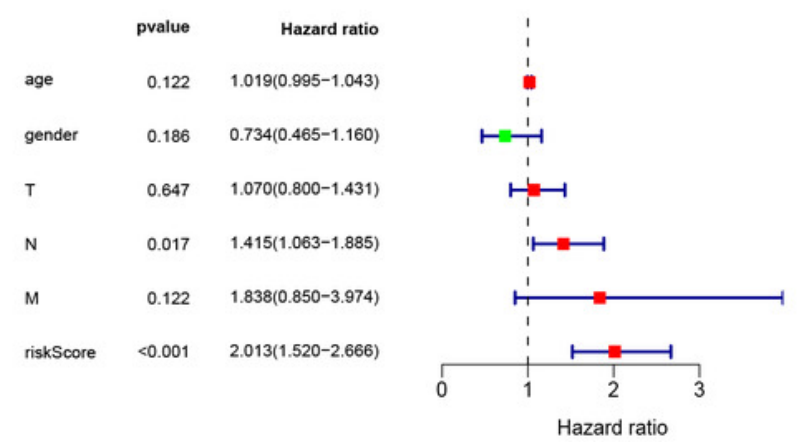




\section{Figure 4}

Development and validation of DNA repair-related signature for survival prediction.

(A-B) The GSE31210 expression data were standardization and distribution. (C-D) The GSE37745 expression data were standardization and distribution. (E) Kaplan-Meier survival curves of overall survival in the DNA repair-related risk score groups in GSE31210 set. (F) Kaplan-Meier survival curves of overall survival in the DNA repair-related risk score groups in GSE37745 set. (G) Multivariate Cox hazard ratio analysis shown the signature risk score and other clinicopathological features related to overall survival of GSE31210. (H) Multivariate Cox hazard ratio analysis shown the signature risk score and other clinicopathological features related to overall survival of GSE37745. gender:0 (female); 1 (male); stage: 1, 2, 3, 4. 
A

GSE31210

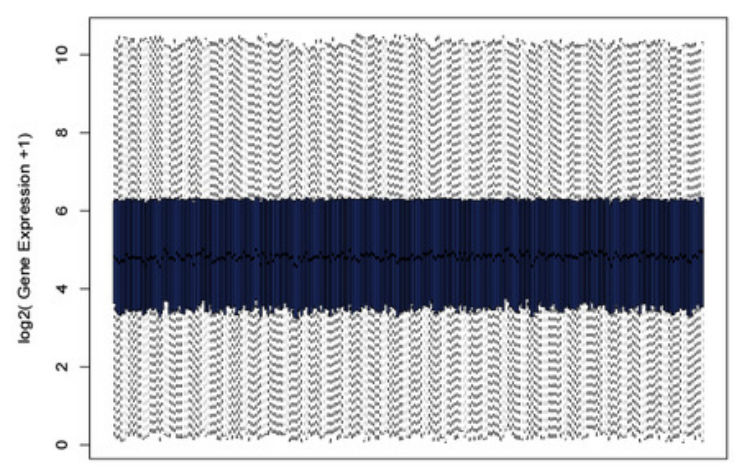

C

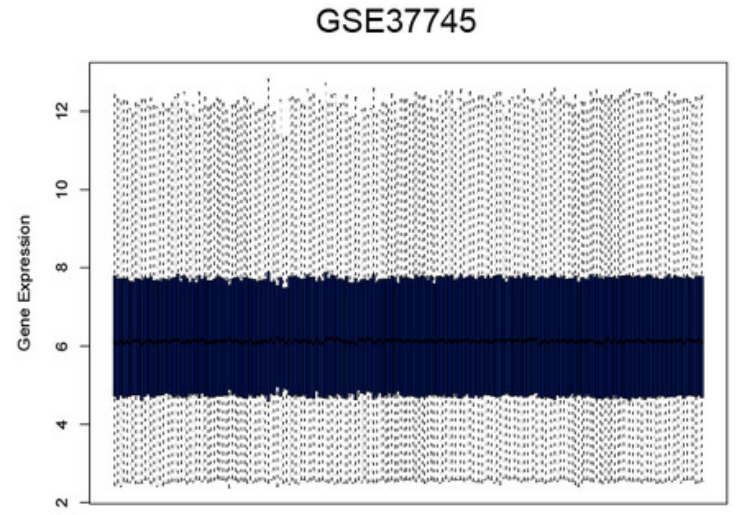

E

Risk \pm High risk $\neq$ Low risk

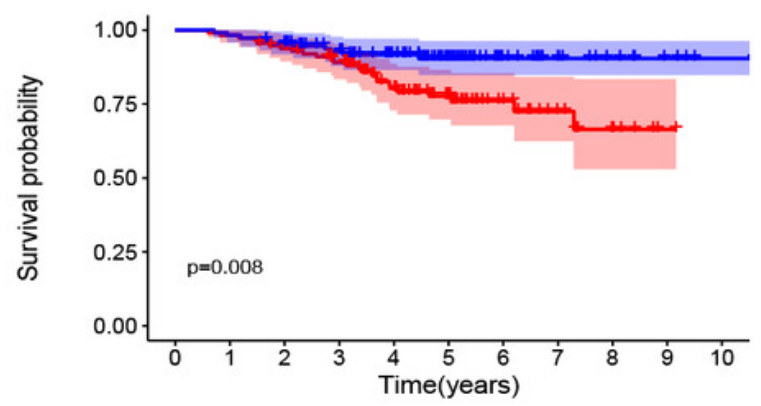

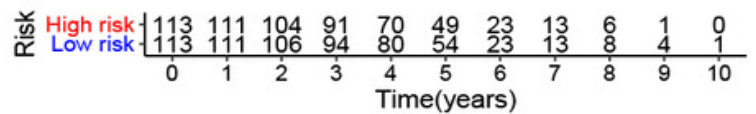

G

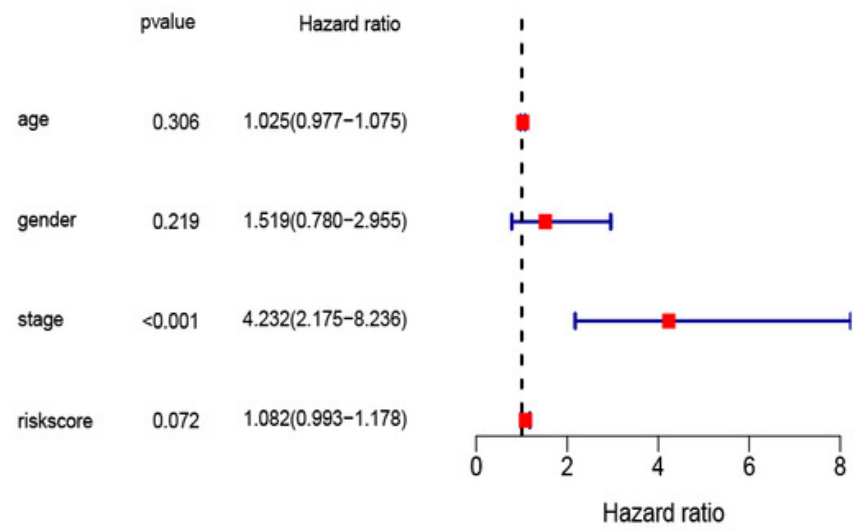

B

GSE31210

D

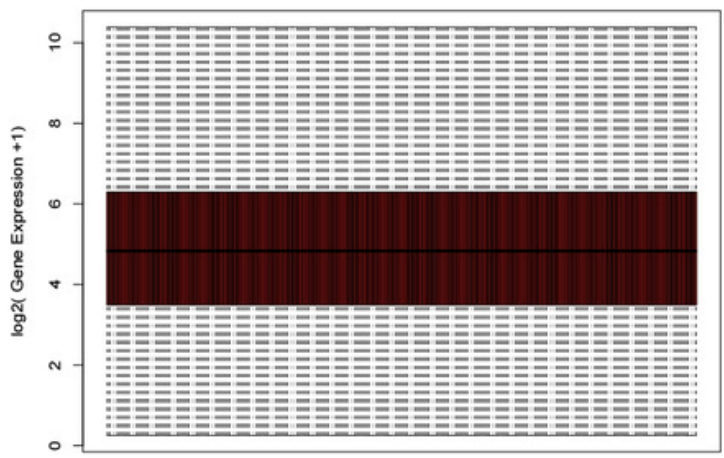

GSE37745

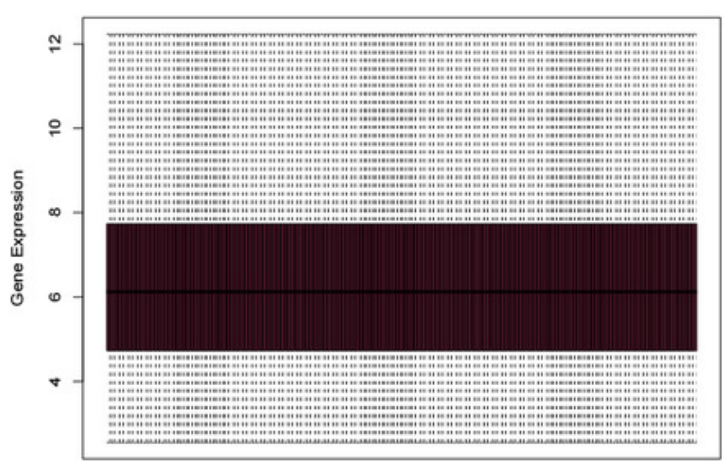

$\mathrm{F}$

Risk $\perp$ High risk $\perp$ Low risk

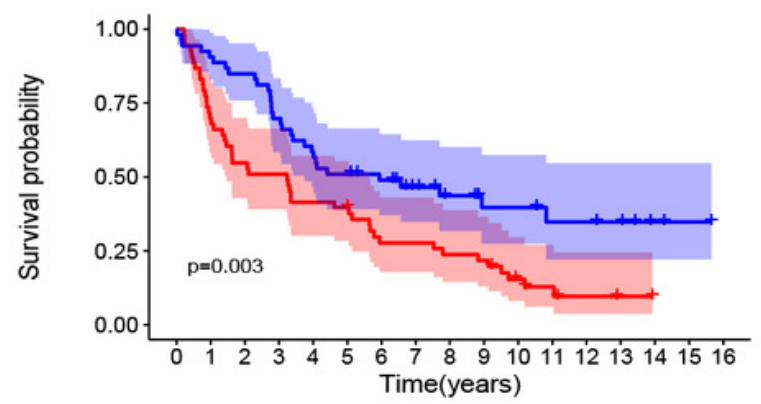

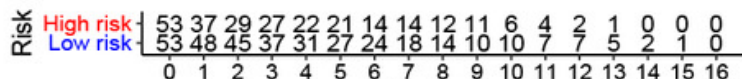
Time(years)

$\mathrm{H}$

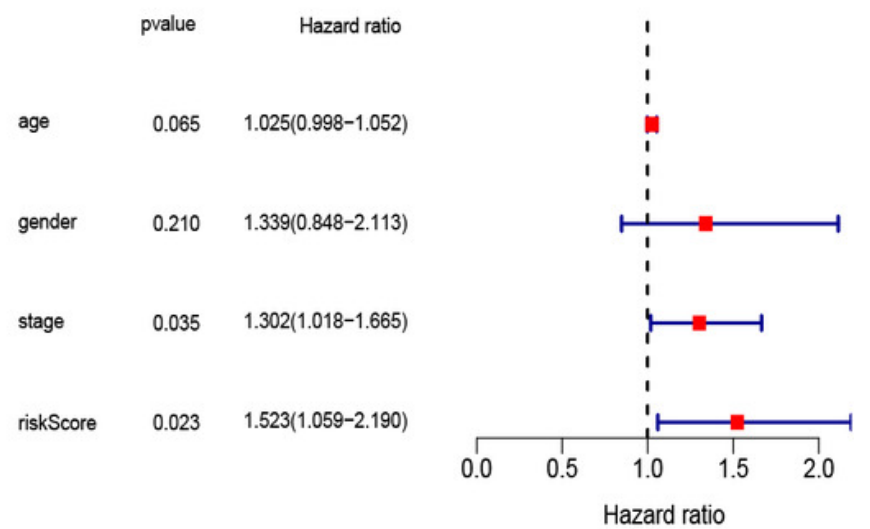


Figure 5

Forest plot of 6 DNA repair-related gene in Osluca online database.

The hazard ratio of each DNA repair-related gene of the signature in the multiple lung cancer datasets (A) PLK1, (B) FOXM1, (C) PTTG1, (D) CCNO, (E) HIST3H2A, (F) BLM.

A
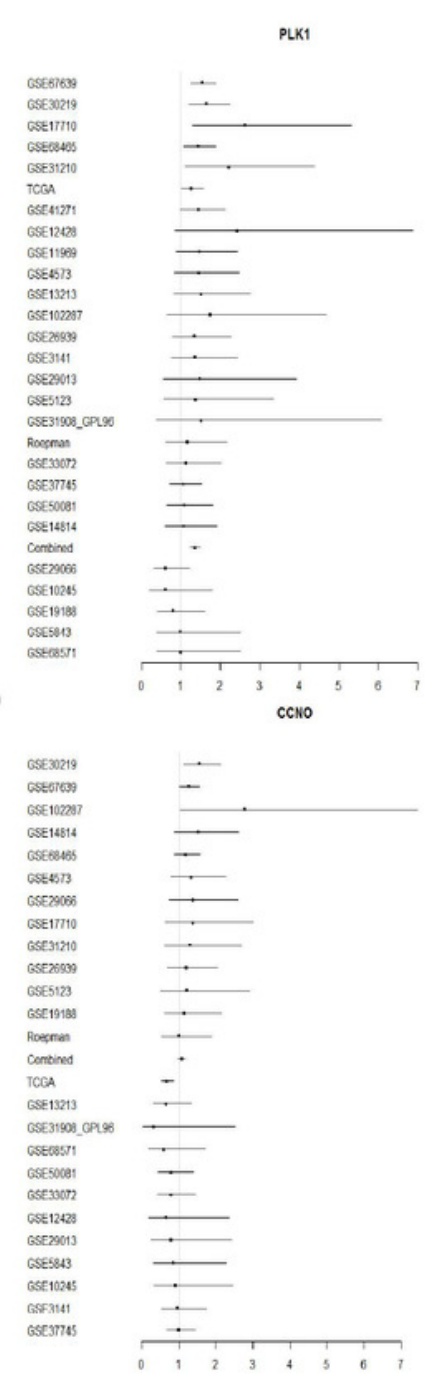

B
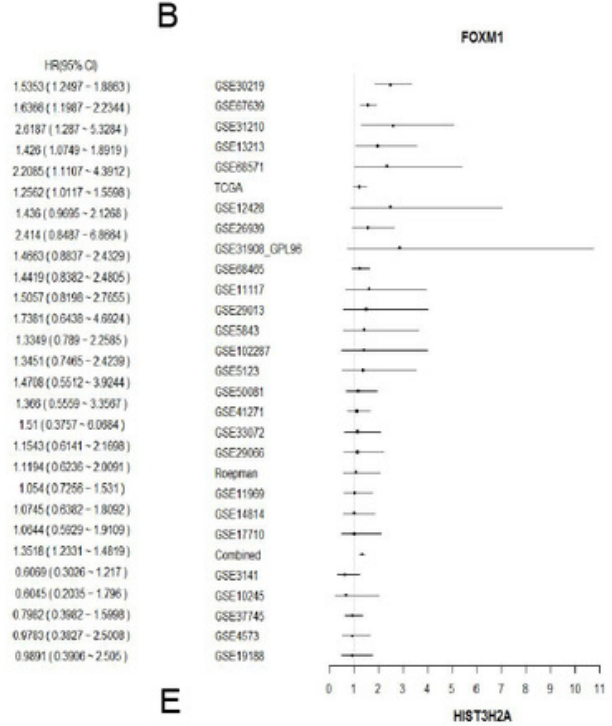

$\sin (1143-21248)$ $1258(102094-1.5035)$ $27861(1054-7499)$ $1.5283(0.8528-26162)$ $111862(008006-15075$ $1.3333(07283-220202$ $1.3821(000255-301003$ $12051(0621-26056)$
$12037(07057-20532)$ $12228(0.5131-2813)$ $1.1000(00000-21004)$ $10045(05008-18503)$

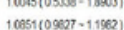

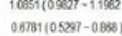
06871 (05287-0836) 0658 (03167- 13458$)$ $03159(00094-25313)$ $0.5872(02503+1.7213)$ $0797(0.4523-10003$ $0.791(0.4344-14005)$

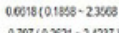
$0.797(0.0251-21237)$ $0.05611034-24582)$ $0.960105274-1745$ $0.0913(060518-1.4413)$
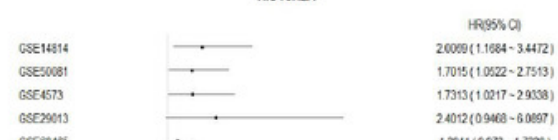

GSECON13

Cose 191930

CSE10245
CSEs1908_CPR

CSEs190_CPLAS
GSE11959

GSE11977

asesi210

asereosos
Aloesnes

Roesnen
Cose31908_CPI97

CSE 102287
GSEs0219

asezoris
aserose

aserecoso
csexson

asexisin

Contions

TOCA
GSE12428

asestat
gessants

Gesemint
HRPS\% CA

28561 (1.8354-3.3066) $15258(1.27-19227)$
$25881(1.3234-5.0011)$ $19884(19599-35545)$

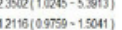
$24580(0.803-7.0199)$ ) 1567 (0953 - 26337) 28485 (07559-10744) $12222(0.02201-1.0503)$ $1612(00641-3.3582)$ $15003(0.053-40101)$ $14293(05622-36308)$ $1.412(04984-40003)$
$13575(05253-35325)$ $1.1580(0.0997+1.5860)$ $11257(0.7576-1.678)$ $11516(0.6320-20065)$ $11166(0.5966-21996)$ 10337 (060076-1.756) 1622 (0 Sesi- - 18065) $104(0.02525-21312)$ $13337(12182-1$ 1628) $0.64(03313-12361)$ $1681(02303-20139)$

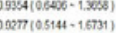
$052404043-1.731)$

[R95\% Ci $1.7015(10522-27513)$ $17313(10227-29208)$ 24012 (0946e-60es) $12911(092-1728)$ 1.5A7 (0835e-2B505) 1.8906(00912 - 41465) $1.6906(00912-41643)$
$20155(05179-78793)$ $20155(05179-78723)$
$12721(0.7612-2125)$ $120 \times 9000006-17765$ 13655(00674-27868) $12078(0068-24123)$ 12986(00060-23567) 1.756r(0.0235-7.55T) 1.4728 (0.509-39083) $1.1025(07 \times 91-15272)$ 1.1324(100567-19528) 1.1059(06128-1968) $10083(0.5778-20878)$ $1.1562(10455-12554)$ $0.8987(0.0038-1.1263)$ 06113(01719-21741) $08235(04504-15067)$ 0.928 (0.04185-1950)
C
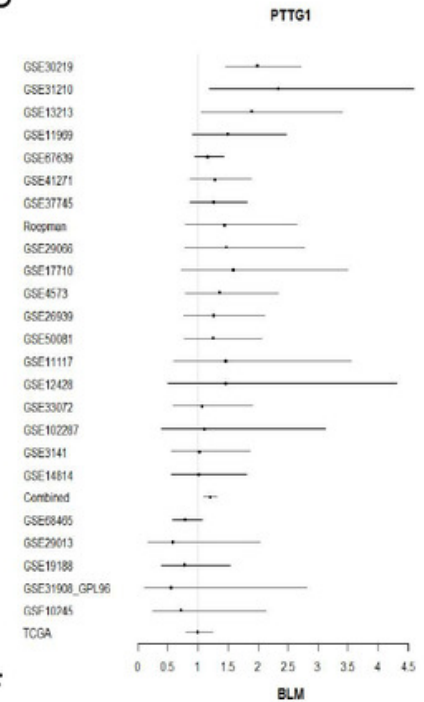

मros a $19864(1.4562-2719)$
$23960(1.1868-40004)$ $23306(1.1608-4.6004)$
$18911(10194-34079)$ (.4911(05583-2475) $11639(09122-14378)$ $12807(08706-18899)$ $12045(08766-18239)$ 1.455(0.7485-20483) $1.4606(07755-2781)$ 13919 (0726s-34975)
$13547(0708-23023)$ $12062(07585-21204)$ 1 ×u 1 1509 (0) 1entosm 19

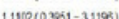
(10005050 $1.201(05889-18281)$
$10105(05820-1813)$ $10105(05625-1813)$
$11978(10912-13147)$

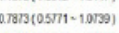
$0.5065(0.1602-20201)$ (a7r8 $(03561-15468)$ (1553)(01807-28159) $07 m$ (0)

F
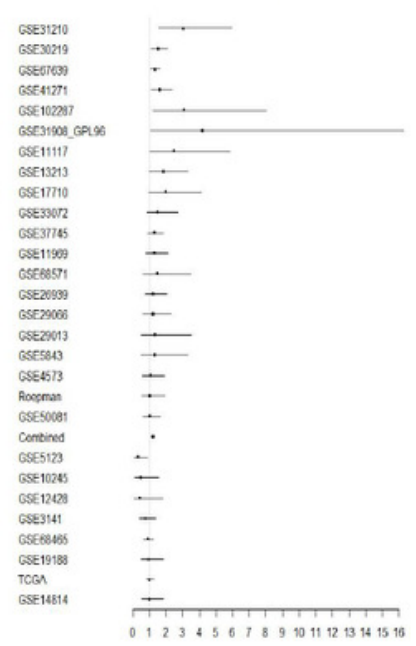

Hados ch $300099(1.50-59280)$
$52861(1.1176-20003)$ $13288(1.0779-10303)$ $16447(1.1054-2.3607)$ $3014(1788-80163)$ 2009(1002-5ens)

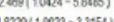

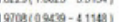
(20) 12775 (0.8985- 18427$)$ $1278(0.755)-21958)$ $1.78(00250-3078)$ $12104607170-20034)$ 12007(00053-22009) $1.3824(04045-3.599)$ $12016(0.5055-3.3003)$ $10658(0.5971-1.5023)$ 10147 (0.507- 19025$)$ $10091(00028-10050)$ $1.1904(1052-13152)$

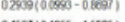
$04587(0.1350-1.283)$

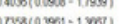

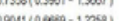
(2) $0.0601(0.0700-1.244)$ $09980(05461-1808)$ 


\section{Figure 6}

Relationship between 6 DNA repair-related gene signature risk score and clinicopathological features.

(A) The relationship between risk score distribution and clinical parameters stratification. Including age, gender, tumor pathologic stage, T stage, $\mathrm{N}$ stage and $\mathrm{M}$ stage in TCGA surgical LUAD. (B) The relationship between risk score distribution and clinical parameters stratification. Including age, gender, tumor pathologic stage and smoking in GSE31210 surgical LUAD. (C) The relationship between risk score distribution and clinical parameters stratification. Including age, gender, and tumor pathologic in GSE37745 surgical LUAD. *Represent for $p$-value $<0.05$; *Represent for $p$-value $<0.01$; NS represent no statistical difference.

A

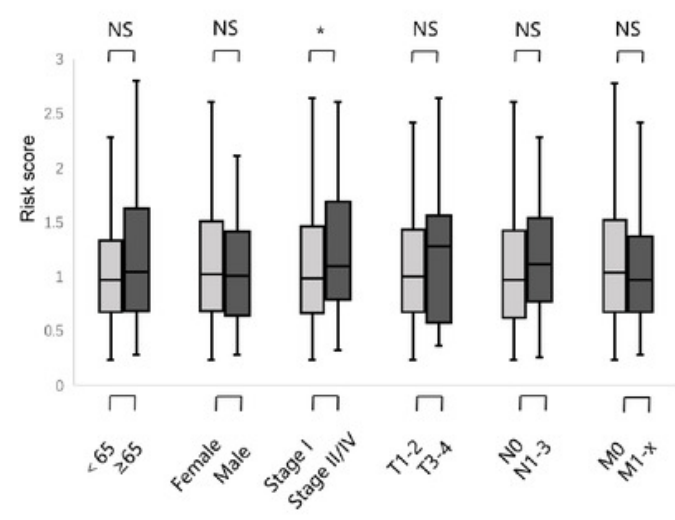

B

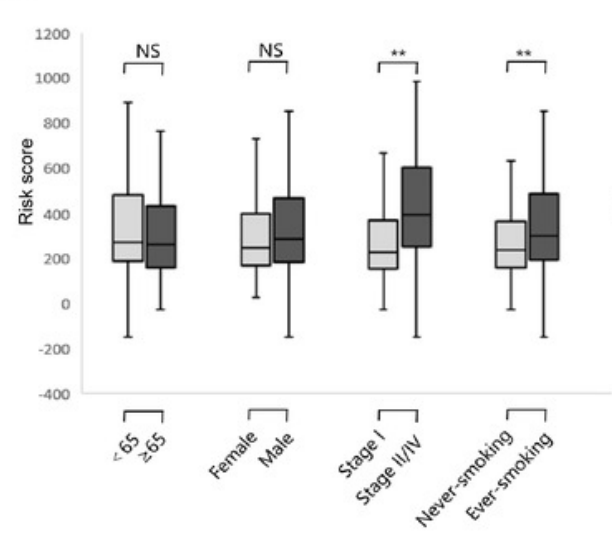

C

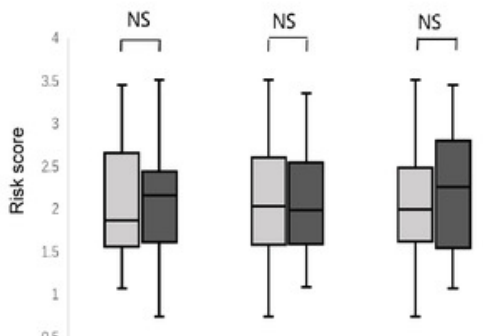

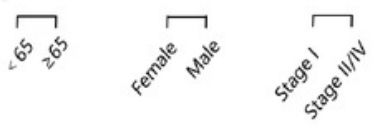


Figure 7

6 gene sets significantly enriched in the surgical LUAD phenotype using GSEA.

Including (A) cell cycle, (B) oocyte meiosis, (C) DNA replication, (D) homologous

recombination, (E) mismatch repair and (F) nucleotide excision repair pathway.

A

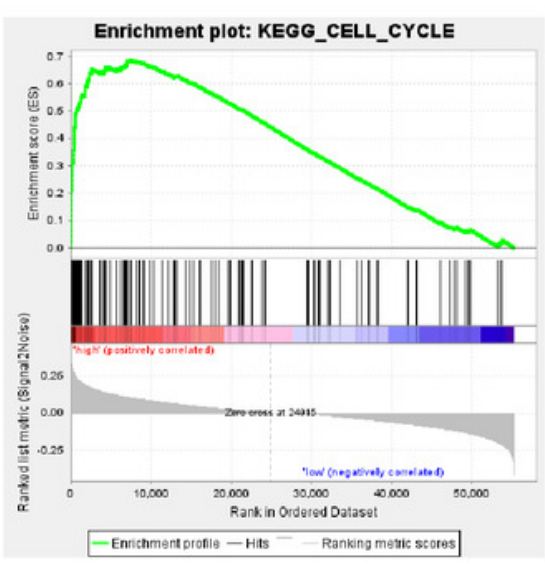

D

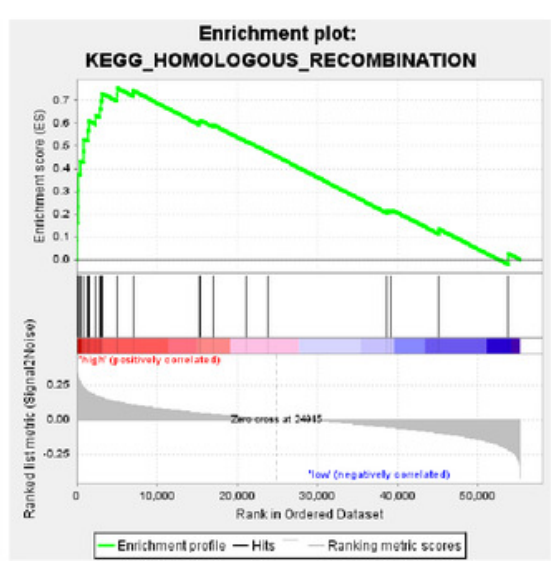

B

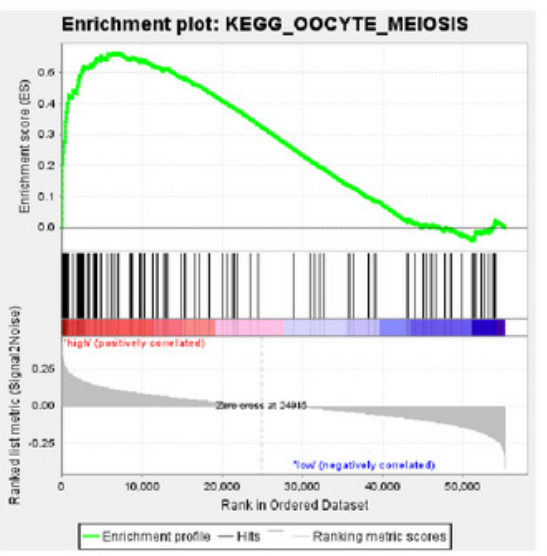

$\mathrm{E}$

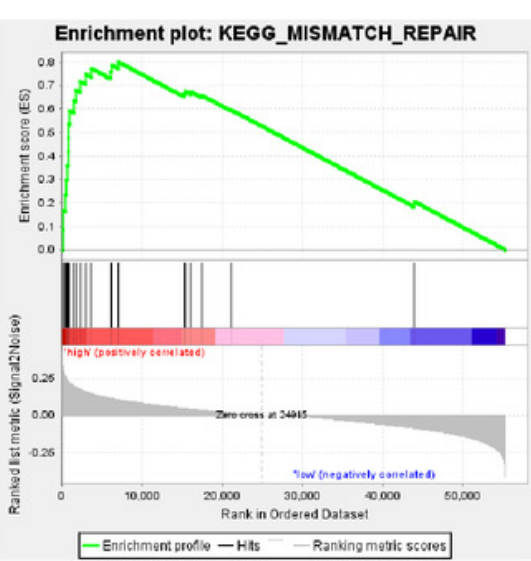

C

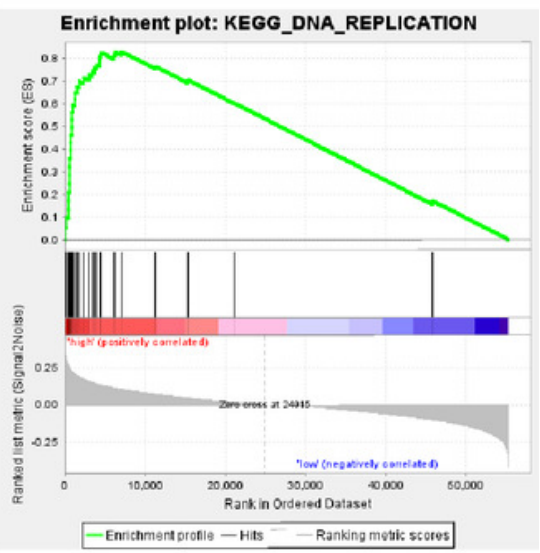

F

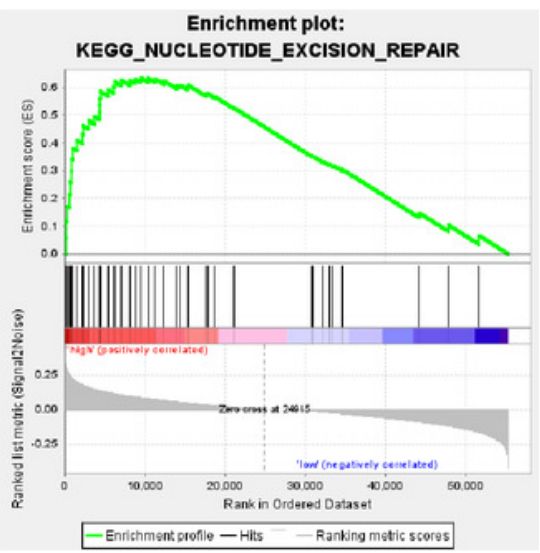

